\title{
RECURSIVE FUNCTIONS DEFINED BY ORDINAL RECURSIONS ${ }^{1}$
}

\author{
R. J. FABIAN AND C. F. KENT
}

A function, $\phi\left(x_{1}, \cdots, x_{n}, y\right)$, will be said to be defined by (un-

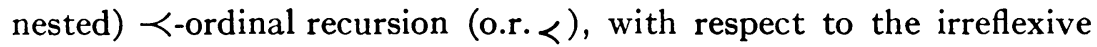
well-ordering $\prec$ of the set of natural numbers if it is defined by a sequence of Kleene's five schemes for primitive-recursive (p.r.) functions [KIM, p. 219] and the ordinal recursion scheme,

$$
\begin{aligned}
& f(\hat{a}, 0)=g(\hat{a}) \\
& f\left(\hat{a}, b^{\prime}\right)=k\left(\hat{a}, b, f\left(\hat{a}, h_{\prec}\left(b^{\prime}, l(\hat{a}, b)\right)\right)\right)
\end{aligned}
$$

where $k, g$ and $l$ denote o.r. $\prec$ functions and we assume that 0 is the least element of $\prec$, and $h \prec$ denotes the function $\theta \prec(b, c)=c$ if $c \prec b$, 0 otherwise.

If the well-ordering $\prec$ is general recursive, by Church's Thesis, the o.r. functions are general recursive. If $\prec$ is the natural order, the o.r. $\prec$ functions are the p.r. functions, and by making $\prec$ a "natural" p.r. well-ordering of type $\omega^{\omega}$, we obtain a function which is not primitive recursive, as is shown, e.g., in Péter's paper, [P 50, p. 271]. By increasing the order type of $\prec$, it is possible to construct an expanding hierarchy of recursive functions where computation difficulty is related to order-type. Hopes of a natural connection between ordertype and computation difficulty were destroyed by results of Routledge [R 53] and Myhill [M 53], which showed that each general recursive function is definable by o.r. $\prec$ for a general recursive wellordering, $\prec$, of type $\omega$ (in Myhill's result, $\prec$ is p.r.). The proof of Myhill's result appears in Liu [L 60].

For a well-ordering (w.o.) of type $\omega$, we use the symbols $\mathrm{pd} \prec$ and $\mathrm{sc} \prec$ to denote the predecessor and successor functions, respectively, on $\prec$. An examination of Liu's w.o., $\prec$, used in the definition by o.r. $\prec$ of the general recursive function, (g.r.), $\phi$, (in Liu's notation) shows that:

$$
\phi\left(x^{\prime}\right)=U\left(\left(\mathrm{sc}_{\prec}\left(2^{x}+\sigma(x, 0)\right)\right)_{1}\right)
$$

Presented to the Society, January 24,1964, under the titles $A$ generalization of Axt's primitive recursive hierarchy, and Reduction of ordinal recursion; received by the editors September 19, 1966.

1 This research was sponsored by the Office of Naval Research under Grant NONR-00038 (63) and forms a part of the Ph.D. thesis of the first author. 
where $\sigma$ is a p.r. function. Hence, while Liu's pd $\prec$ is p.r., $\phi$ is primitive recursive in $\mathrm{sc} \prec$, and thus $\mathrm{sc} \prec$ cannot generally be p.r. This is the basis of a comment at the end of the paper [L 62], attributed to Hartley Rogers, Jr., that the recursive complexity of $\phi$ is "absorbed" by $\mathrm{sc} \prec$. In this paper, we show that it need not be true that sc $\prec$ "absorbs" the complexity of $\phi$ by duplicating the Myhill-Liu result for a p.r.w.o. of type $\omega$, for which both $\mathrm{pd} \prec$ and sc $<$ are also p.r. This additional structure further makes it possible to duplicate Liu's "generalized concept" of primitive recursion [L 62 ] under conditions where the permutation, $\mathrm{pm} \prec$, and the successor function, $\mathrm{sc} \prec$, used there, are both primitive recursive. In addition, since our constructions are very simple, we shall not always produce p.r. defining equations for functions and predicates asserted to be p.r. We use Greek letters and mnemonic Latin combinations like pd, sc, for informally defined functions. Script Latin letters are used as function letters in recursion schemes only.

THEOREM 1. For each general-recursive function $\phi$, (temporarily of one variable) there exists a p.r.w.o., $\prec$, of type $\omega$, with both $\mathrm{pd} \prec$ and $\mathrm{sc}<p . r$., and so that $\phi$ is o.r. $\prec$.

Proof. Let $g$ be a fixed Gödel number for $\phi$, and let $\delta(n)=2 n+1$. Any 1-1 increasing p.r. function could be used in place of $\gamma$. We choose $\tau(x, y), \pi_{1}(z), \pi_{2}(z)$ as names for a triple of primitive recursive pairing and projection functions, $(\forall z)\left(\tau\left(\pi_{1}(z), \pi_{2}(z)\right)=z\right)$, subject to the restrictions $(\forall z)\left(z \geqq \pi_{2}(z)\right)$ and $\tau(0,0)=0$. Further, we denote $\tau(x, y)$ by $\langle x, y\rangle$. Clearly, if we can construct $\prec$ so that the following condition, $C$, is satisfied,

$C: \quad$ For each $n:\left\langle\mu z T_{1}(g, n, z), \gamma(n)\right\rangle \prec\left\langle\mu z T_{1}(g, n, z) \div 1, \gamma(n)\right\rangle$ $\prec \cdots \prec\langle 1, \gamma(n)\rangle \prec\langle 0, \gamma(n)\rangle$

then, just as Liu does, we can use the o.r. $\prec$ scheme.

$$
\begin{aligned}
f(0)= & 0 \quad \text { if } \\
f\left(b^{\prime}\right)= & f\left(h<\left(b^{\prime},\left\langle p_{1}\left(b^{\prime}\right)+1, p_{2}\left(b^{\prime}\right)\right\rangle\right)\right) \quad \text { if } \\
& \quad \mu z T_{1}\left(g, \mu w_{w \leq z}\left(g(w)=p_{2}\left(b^{\prime}\right)\right), z\right)>p_{1}\left(b^{\prime}\right), \\
= & U\left(p_{1}\left(b^{\prime}\right)\right) \quad \text { if } \\
& \quad \mu z T_{1}\left(g, \mu w_{w \leq b^{\prime}}\left(g(w)=p_{2}\left(b^{\prime}\right)\right), z\right)=p_{1}\left(b^{\prime}\right), \\
= & 0 \quad \text { otherwise. }
\end{aligned}
$$

(NB. The defining clauses in this definition by cases are p.r. and the letters $p$ and $d$ denote the functions $\pi$ and $\delta$ respectively.) The func- 
tion $\Psi$ defined by this scheme will be o.r. $\prec$, by condition C, and $(\forall x)(\phi(x)=\Psi(\langle 0, \gamma(x)\rangle))$. Hence, $\phi$ is o.r. $<$.

It remains to show that there does indeed exist a p.r.w.o., $\prec$, of type $\omega$, satisfying condition $C$. We base our description upon the following fundamental interval for $n, I_{n}$.

$$
I_{n}\left\{\begin{array}{l}
\langle 0,2 n\rangle \prec\langle 1,2 n\rangle \prec \cdots \prec\left\langle\mu z T_{1}(g, n, z), 2 n\right\rangle \\
\prec\left\langle\mu z T_{1}(g, n, z), 2 n+1\right\rangle \prec\left\langle\mu z T_{1}(g, n, z)-1,2 n+1\right\rangle \\
\prec \cdots \prec\langle 1,2 n+1\rangle \prec\langle 0,2 n+1\rangle .
\end{array}\right.
$$

Let $I=\bigcup_{n=0}^{\infty} I_{n}$. We construct the order $\prec$ as follows:

$$
I_{0} \prec\left(S_{0}\right) \prec I_{1} \prec\left(S_{1}\right) \prec \cdots \prec\left(S_{n-1}\right) \prec I_{n} \prec\left(S_{n}\right) \prec \cdots
$$

where the "space" $\left(S_{n}\right)$ is filled by the number $n$ if $n \notin I$, and left vacant otherwise. It is easily seen that the predicate $z \in I$ is primitive recursive and, hence, the ordering $\prec$ and the functions $\mathrm{pd} \prec, \mathrm{sc} \prec$ are all primitive recursive.

The restriction in Theorem 1 to functions of a single variable is immediately removable in the usual manner, i.e. given $\phi\left(x_{1}, \cdots, x_{n}\right)$, define $\phi^{\prime}(x)=\phi\left((x)_{1}, \cdots,(x)_{n}\right)$; apply Theorem 1 to $\phi^{\prime}$ and immediately, $\phi$ is o.r. $\prec$. With Liu, we let $\mathrm{pm} \prec$ denote the permutation of the natural numbers which maps the natural order into the order $\prec$, for a w.o. of type $\omega$. The permutation $\mathrm{pm} \prec$ has the property:

$$
(\forall x)(\forall y)\left(x<y \Leftrightarrow \mathrm{pm}_{\prec}(x) \prec \mathrm{pm} \prec(y)\right) .
$$

Since $\mathrm{pm} \prec(0)=(0), \mathrm{pm} \prec\left(x^{\prime}\right)=\mathrm{sc}^{\left(x^{\prime}\right)}(0)$, we see that $\mathrm{pm} \prec$ is primitive recursive in $\mathrm{sc} \prec$. Hence, we have the following corollary.

COROLlary 2. The permutation, $\mathrm{pm} \prec$, associated with the wellordering of Theorem 1, is primitive recursive.

Thus Liu's "generalized primitive recursion" can be accomplished with $\mathrm{pm} \prec$ and $\mathrm{sc} \prec$ both primitive recursive. If both $\mathrm{pm}<$ and its inverse, $(\mathrm{pm} \prec)^{-1}$, are primitive recursive, then, clearly, $\phi$ is also primitive recursive. More generally, we have the following theorem, which is quite easy to prove, and which shows that the recursive complexity of $\phi$ is now "absorbed" by $(\mathrm{pm} \prec)^{-1}$, although this function makes no explicit entry into Liu's "generalized" primitive recursion for $\phi$.

THEOREM 3. For any well-ordering of type $\omega, \prec$, and function $\phi$ which is o.r. $<, \phi$ is p.r. in $(\mathrm{pm} \prec)^{-1}$.

Proof. We use induction on the length of the o.r. $\prec$ description 
of $\phi$. Only the induction step is nontrivial and this only in case the o.r. $\prec$ ends in an application of the scheme

$$
f(\hat{a}, 0)=g(\hat{a}) \quad f\left(\hat{a}, b^{\prime}\right)=k\left(\hat{a}, b, f,\left(\hat{a}, h \prec\left(b^{\prime}, l(\hat{a}, b)\right)\right)\right)
$$

where, the functions $\gamma, \kappa, \lambda$, represented by $g, k, l$ are o.r. $\prec$ and, by induction, p.r. in $(\mathrm{pm} \prec)^{-1}$. By Theorem 6 of Routledge [R 53], $\phi$ is p.r. in $\gamma, \kappa, \lambda$ and in

$$
\Psi(y)=\mu z\left(\theta^{(z)}(y, \lambda(x, y))=0\right)
$$

where $\theta(z) \prec$ represents the $z$ th element of the splinter of $\theta \prec$ (zth iteration of the function), beginning with $y$. Since $\theta<$ is clearly p.r. in $(\mathrm{pm} \prec)^{-1}$, and $\Psi(y) \leqq\left(\mathrm{pm}_{\prec}\right)^{-1}(y)$, we see that $\phi$ is p.r. in $(\mathrm{pm} \prec)^{-1}$.

In his review of [L 62], Myhill [M 66], asks whether there is a primitive recursive well-ordering of type $\omega, \prec$, for which $\mathrm{sc} \prec$ is not even general recursive. Such orderings are easy to construct and, in fact, Markwald, [Ma 54], has shown how to construct such orders with neither $\mathrm{pd} \prec$ nor $\mathrm{sc} \prec$ general recursive. Augmenting Markwald's result, we can prove the following theorem. Note that if either $\mathrm{pd} \prec$ or $\mathrm{sc} \prec$ is general recursive, then $\prec$ is also general recursive, since $\prec$ is of type $\omega$.

Theorem 4. For each of (i), (ii), (iii), below, it is possible to find a general recursive well-ordering, $\prec$, of the natural numbers having type $\omega$, for which one and only one of (i), (ii), (iii), is primitive recursive.

(i) the order predicate $\prec$,

(ii) $\mathrm{pd} \prec$,

(iii) $\mathrm{sc}<$.

Proof. We prove only the case sc $\prec$ p.r., $\prec$, and pd $\prec$ not p.r. The symmetric case has a symmetric proof and the third, with $\prec$ p.r., is Markwald's result. We shall assume that the characteristic function of the order predicate $\prec$ is a function $\chi \prec$ with the property

$$
x \prec y \Leftrightarrow \chi_{\prec}(x, y)=0 .
$$

We construct a g.r.w.o., $\prec$ of type $\omega$, for which $\mathrm{sc} \prec$ is p.r. by diagonalizing over the p.r. functions of two variables. To this end, let $\eta(n)$ be a p.r. function whose range is a set of Gödel numbers for two-placed primitive recursive functions and contains at least one Gödel number for each such function. For each $n$, we define two intervals

$$
\begin{aligned}
& A_{n}: \quad\langle 0, n\rangle \prec\langle 2, n\rangle \prec \cdots \prec\left(2 m_{n}, n\right\rangle \\
& B_{n}: \quad\langle 1, n\rangle \prec\langle 3, n\rangle \prec \cdots \prec\left\langle 2 m_{n}-1, n\right\rangle
\end{aligned}
$$


where:

$$
m_{n}=2^{\mu_{n} 3^{\mu_{n}+1}} \quad \text { and } \quad \mu_{n}=\mu z T_{2}(\eta(n),\langle 0, n\rangle,\langle 1, n\rangle, z) .
$$

Let $C=\bigcup_{n=0}^{\infty} A_{n} \cup \cup_{n=0}^{\infty} B_{n}$ and let $I_{n}$ be the interval $A_{n} \prec\left(S_{n}\right) \prec B_{n}$ if $U\left(m_{n}\right)_{1} \neq 0$ and $B_{n} \prec\left(S_{n}\right) \prec A_{n}$ if $U\left(m_{n}\right)_{1}=0$. Again, the space $\left(S_{n}\right)$ is filled by the number $n$ if $n \notin C$ and is left blank otherwise. The predicate $n \in C$ is primitive recursive and this, together with the fact that the decision whether $A_{n+1} \prec B_{n+1}$ or $B_{n+1} \prec A_{n+1}$ can be made primitive recursively in the last member of $I_{n}$ shows that the successor function $\mathrm{sc} \prec$ is primitive recursive.

The diagonal construction shows that the order predicate, $\prec$, is not primitive recursive, for define

$$
\begin{aligned}
\Psi(x, y) & =0 & & \text { if }\langle 0, x\rangle \prec\langle 1, y\rangle \\
& =1 & & \text { otherwise }
\end{aligned}
$$

if $\prec$ were p.r., then $\Psi(x, y)$ would be a p.r. function of two variables and we could choose $n$ so that $\eta(n)$ is a Gödel number of $\Psi$. Then

$$
\begin{aligned}
\{\eta(n)\}(\langle 0, n\rangle,\langle 1, n\rangle)=\Psi(n, n) & =0 \\
& \Leftrightarrow U\left(m_{n}\right)_{1}=\{\eta(n)\}(\langle 0, n\rangle,\langle 1, n\rangle) \neq 0 .
\end{aligned}
$$

This contradiction shows that $\prec$ is not p.r., and $\Psi(x, x)$ is not p.r. Finally, since

$$
\Psi(x, x)=0 \Leftrightarrow \operatorname{pd}_{\prec}(\langle 1, x\rangle)=x \vee p_{2}\left(\operatorname{pd}{ }_{\prec}(\langle 1, x\rangle)\right)=x
$$

then $\mathrm{pd} \prec$ cannot be p.r. without making $\Psi(x, y)$ primitive recursive, and hence, $\mathrm{pd} \prec$ is not p.r.

\section{REFERENCES}

[KIM] S. Kleene, Introduction to metamathematics, Van Nostrand, Princeton, N. J., 1952.

[L 60] S. Liu, $A$ theorem on general recursive functions, Proc. Amer. Math. Soc. 11 (1960), 184-187.

[L 62] - A generalized concept of primitive recursion and its application to deriving general recursive functions, Hung-Ching Chow Sixtieth Anniversary Volume, Inst. of Math., Acad. Sinica, Taipei, 1962, pp. 93-98.

[Ma 54] W. Markwald, Zür Theorie der konstrucktive Wohlordnung, Math. Ann. 127 (1954), 135-149.

[M 53] J. Myhill, A stumbling-block in constructive mathematics, J. Symbolic Logic 18 (1953), 190-191.

[M 66] — Review of [L 62], Math. Reviews 31 (1966), 51.

[P 50] R. Péter, Zusammenhang der mehrfachen und transfiniten Rekursion, J. Symbolic Logic 15 (1950), 248-272.

[R 53] N. Routledge, Ordinal recursion, Proc. Cambridge Philos. Soc. 49 (1953), 175-182.

\section{Smith College and}

Case Western Reserve University 